\title{
Effects of miR-99a on the migration and proliferation of glioma cells
}

\author{
Yifan Xu, Tianyu Lu, Wu Xu, Yuxiang Dai, Weibang Liang*, Wei Jin \\ Nanjing Drum Tower Hospital Jiangsu, China
}

\begin{abstract}
Background: To evaluate the effects of miR-99a on the migration and proliferation of glioma cells.

Materials and Methods: Glioma cell line LN229 with stable up-regulation of miR-99a was constructed by transfection of hsa-miR-99a mimics, and cells with stable miR-99a knock-down were established by transfection of hsa-miR-99a inhibitor. The proliferation capacities of two groups were detected by the MTT assay, and their migration capacities were detected by the scratch assay.

Results: LN229 cells with stable up-regulation and knock-down of miR-99a were successfully constructed. Up-regulating miR-99a inhibited the proliferation and migration of glioma cells, but knocking down this gene promoted their proliferation and migration.

Conclusion: MiR-99a significantly affected the proliferation and migration of glioma cells, as a potentially eligible target for glioma therapy.
\end{abstract}

Keywords: miR-99a, migration, proliferation, glioma

Received: $21^{\text {st }}$ August 2017; Accepted: 10 ${ }^{\text {th }}$ January 2018; Published: $15^{\text {th }}$ April 2018

\section{Introduction}

Glioma is the most common primary tumor of the central nervous system, accounting for about $40 \%$ of all intracranial tumors. Particularly, the annual incidence rate of malignant glioma is about $3-8 / 100,000$ population [1,2]. To date, the etiology or pathogenesis of glioma remains unclear. Glioma is usually accompanied by overexpression of one or more related oncogenes. In addition, it may also be associated with mutation and deletion of some anti-oncogenes. A variety of genetic factors synergistically induce abnormalities in cellular signaling pathways, and tumor cells undergo autonomous invasion and proliferation after escaping from the normal growth regulatory system, thus producing relevant malignant phenotypes [3]. Recently, miR99a has been reported to predominantly control the onset and progression of many cancers including tongue squamous cell carcinoma, lung cancer, kidney cancer, bladder cancer, ovarian cancer, hepatocellular carcinoma, pediatric adrenal carcinoma, and prostate cancer [4]. For example, Zhang et al. [5] found in 152 primary hepatocellular carcinoma samples that 111 cases

*Corresponding author: Weibang Liang, Nanjing Drum Tower Hospital, Nanjing, China.

E-mail: liangweibangdn@sina.com 
$(73.03 \%)$ had significantly lower miR-99a expressions than those of normal subjects, which were correlated with low tumor differentiation, liver cirrhosis, tumor-free survival rate and overall survival rate. By performing RT-PCR, Feng et al. [6] found that miR-99a was lowly expressed in 7 bladder cancer cell lines and 100 bladder cancer tissue samples. Meanwhile, low miR-99a expression was correlated with the aggressive phenotype of bladder cancer, whereas miR-99a overexpression significantly inhibited the growth of tumor cells and arrested them in the G1/S phase. Moreover, the plasma of patients with bladder cancer also had low miR-99a expression.

It has been confirmed that $[7,8]$ miR-99a, as an anti-oncogene, was down-regulated in a variety of tumors, thus participating in the proliferation, differentiation, apoptosis and other growth regulatory processes of tumor cells. However, the role of miR-99a in glioma has seldom been referred. Only Parker et al. [9] have reported that glioma had high miR-99a expression level. However, a carcinogenic fusion gene FGFR3-TACC3 formed in glioma, which induced the deletion of 3'-UTR from FGFR3 gene, hindering miR-99a from exerting regulatory effects. To date, how miR-99a regulates glioma cells has never been studied.

Lentiviral vector is a gene vector derived from HIV-1 research. It has many advantages, such as broad spectrum of infection, effective infection of both dividing and resting cells, and long-term stable expressions of exogenous genes. Currently, lentivirus systems have been widely applied in the gene overexpression, RNA interference and miR studies of various tumor cell lines as well as in vivo animal experiments. To evaluate the effects of miR-99a on the growth phenotype of glioma cells, we explored its roles in the proliferation and migration of glioma cell line LN229 through gene up-regulation and knock-down.

\section{Materials and methods}

\section{Glioma cell line}

Glioma cell line LN229 was purchased from the cell bank of Shanghai Institute of Biochemistry and Cell Biology, Chinese Academy of Sciences (China). Cell identification, authentication, and mycoplasma testing were performed by an expert in the Department of Oncology of our hospital.

\section{Materials}

DMEM, penicillin, streptomycin, $10 \%$ fetal bovine serum (FBS), Lipofectamine ${ }^{\mathrm{TM}} 2000$ reagent, and $0.25 \%$ trypsin were purchased from Gibco (USA). All lentiviruses were designed and synthesized by Shanghai GenePharma Co., Ltd. (China), and LV3 was used as the interference vector. Sequences for nonsense control vector LV3NC: 5'-TTCTCCGAACGTGTCACGT-3'; has-miR-99a mimics: 5'-AACCCGTAGATCCGATCTTGTG-3'; has-miR-99a inhibitor: 5'-CACAAGATCGGATCTACGGGTT-3'. HasmiR-99a inhibitor blocked the translation of miR-99a mRNA by binding its 3' untranslated region through complete or incomplete base pairing, thereby inhibiting the expression of miR-99a. On the other hand, has-miR-99a mimics was mainly added at the upstream of miR99a to markedly promote its transcription and translation, finally inducing overexpression.

\section{Cell culture}

LN229 cells were added in DMEM containing $10 \%$ FBS, $1 \%$ penicillin and $1 \%$ streptomycin, and cultured in an incubator at $37^{\circ} \mathrm{C}$ with $5 \% \mathrm{CO}_{2}$ and saturated humidity. When the confluence reached about $85 \%$, the cells were passaged.

\section{Cell transfection}

LN229 cells were digested, counted, incubated in 6-well plates at a density of $2.5^{\prime} 10^{5}$ cells/well and cultured in an incubator at $37^{\circ} \mathrm{C}$ 
with $5 \% \mathrm{CO}_{2}$ and saturated humidity. When the confluence reached $80 \%-90 \%$, the cells were transfected with has-miR-99a mimics or hasmiR-99a inhibitor according to the instructions of Lipofectamine ${ }^{\mathrm{TM}} 2000$ reagent. After the transfected cells were cultured for $4 \mathrm{~h}$, the medium was replaced with complete culture medium, and the cells with stable gene up-regulation or knock-down were obtained through puromycin screening and passage. The miR-99a expression in cells emitting green fluorescence was observed under a fluorescence microscope.

\section{Real-time PCR}

Stably transfected LN229 cells were suspended, centrifuged, and transferred to a clean $1.5 \mathrm{ml}$ EP tube by a micropipette. Then $1 \mathrm{ml}$ of Trizol reagent was added to digest the cells at low temperature for about $10 \mathrm{~min}$. Afterwards, $0.2 \mathrm{ml}$ of chloroform solution was added, and the EP tube was sealed and vigorously shaken for 15-30 s, left still for 2-3 min, and centrifuged at $4^{\circ} \mathrm{C}$ and 12000 'g for $15 \mathrm{~min}$. The upper layer was transferred to another clean $1.5 \mathrm{ml} \mathrm{EP}$ tube, added $0.5 \mathrm{ml}$ of isopropanol, mixed and left still on ice for $10 \mathrm{~min}$. The solution was centrifuged at $4^{\circ} \mathrm{C}$ and 12000 ' $\mathrm{g}$ for $15 \mathrm{~min}$, and the precipitate was collected as RNA sample. The RNA sample was thereafter dissolved by adding $20 \mu \mathrm{l}$ of DEPC-water and stored in a $-80^{\circ} \mathrm{C}$ refrigerator. Subsequently, the RNA sample $(0.05 \mathrm{mg})$ was subjected to reverse transcription by using TaqMan MicroRNA reverse transcription kit (Applied Biosystems, USA). Real-time PCR was conducted with 7500 FAST real-time PCR system (Applied Biosystems, USA) and TaqMan universal PCR master mix (Applied Biosystems, USA). The results were corrected by U6RNA internal reference, and the relative expression of miR-99a was expressed as $2^{-\Delta \Delta C t}$. Three independent experiments were carried out. Amplification primer sequences of miR-99: Forward primer: 5'-CATTACTAAACCCGTAGATCCGAT-3', reverse primer: 5'-TATGGTTTTGACGACTGTGTGAT-3'.

\section{Detection of cell proliferation by MTT as-}

say

MTT assay was employed to test the cell viability. MTT solution ( $5 \mathrm{mg} / \mathrm{ml}$ ) was prepared by using PBS or normal saline. LN229 cells (hsamiR-99a mimics group, hsa-miR-99a inhibitor group and NC group) were digested and counted, and the density was adjusted to $1.5 \times 10^{5}$ cells/ $\mathrm{mL}$. After incubation in six-well plates for $24 \mathrm{~h}$, the cells were digested, centrifuged, adjusted to a final concentration of $1 \times 10^{5}$ cells $/ \mathrm{ml}$ and seeded into 96-well plates, $100 \mu \mathrm{L}$ per well. MTT assay was conducted at $0 \mathrm{~h}, 24 \mathrm{~h}, 48 \mathrm{~h}, 72 \mathrm{~h}, 96 \mathrm{~h}$ and $120 \mathrm{~h}$, respectively, and the optical density at $490 \mathrm{~nm}$ was determined by a microplate reader.

\section{Detection of cell migration by scratch as-}

say

LN229 cells (hsa-miR-99a mimics group, hsa-miR-99a inhibitor group and NC group) were cultured for $24 \mathrm{~h}$, digested with trypsin, and then pipetted into a single cell suspension, with the density adjusted to $4 \times 10^{5} / \mathrm{ml}$. The cells were seeded in 6-well plates, $2 \mathrm{ml}$ per well. When the confluence reached $80-90 \%$, a $10 \mu \mathrm{l}$ pipette tip was used to make straight lines evenly in the 6-well plates, and the detached cells were washed off with PBS. Afterwards, the remaining cells were cultured in medium containing $1 \%$ serum. Three independent experiments were performed for each group. Five visual fields in the scratched region were randomly selected and photographed under a microscope $\left(100^{\prime}\right)$ at 0 and $24 \mathrm{~h}$ respectively. The width or acellular area of scratch at each time point was analyzed by Image J software.

\section{Statistical analysis}

All data were analyzed by GraphPad 5.0 software. All experiments were performed in 
triplicate, and the results were expressed as mean \pm standard deviation. $\mathrm{P}<0.05$ was considered statistically significant.

\section{Results}

\section{Construction of glioma cells with stable miR-99a up-regulation or knock-down}

Forty-eight hours after transfection with hsa-miR-99a mimics or hsa-miR-99a inhibitor, the cells with positive expressions emitted green fluorescence. As suggested by the fluorescence intensity, miR-99a expression was significantly up-regulated in the hsa-miR-99a mimics group but significantly down-regulated in the hsamiR-99a inhibitor group compared with that of the NC group (Figure 1). Consistently, RT-PCR showed significantly higher miR-99a expression in the hsa-miR-99a mimics group but significantly lower expression in the hsa-miR-99a inhibitor group than that of the NC group (Figure 2).
Therefore, LN229 cells with stable up-regulation and knock-down of miR-99a had been successfully constructed.

\section{Cell proliferation detected by MTT assay}

MTT assay was used to detect cell proliferation inhibition through measuring the absorbance of formazan dissolved by adding dimethyl sulfoxide to indirectly reflect cell number. Uncontrollable cell proliferation is one of the important malignant phenotypes of glioma. The MTT assay showed that with increasing time of transfection ( $0 \mathrm{~h}, 24 \mathrm{~h}, 48 \mathrm{~h}, 72 \mathrm{~h}, 96 \mathrm{~h}, 120 \mathrm{~h})$, the absorbance gradually rose (Figure 3). Compared with the NC group at $72 \mathrm{~h}$ and $96 \mathrm{~h}[(0.66 \pm 0.11)$ and (1.07 \pm 0.19$)$ respectively], the hsa-miR-99a inhibitor group had significantly higher absorbances $[(1.26 \pm 0.15)$ and $(1.56 \pm 0.16)$ respectively] $(\mathrm{P}<0.05)$. At $24 \mathrm{~h}$, the growth of the hsamiR-99a mimics group was significantly inhibited compared with that of the NC group, which

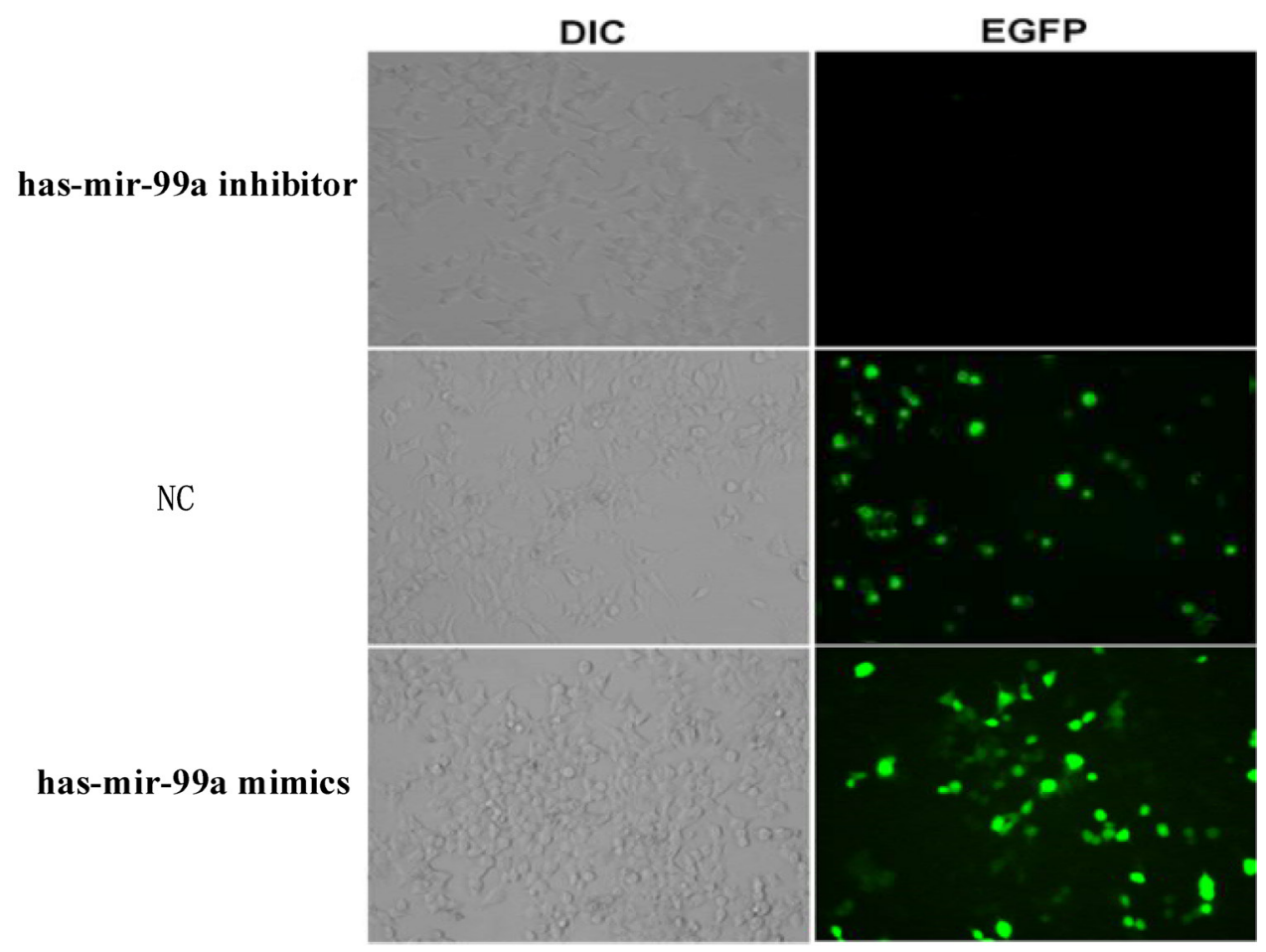

Figure 1. Transfection results of LN229 cells. 


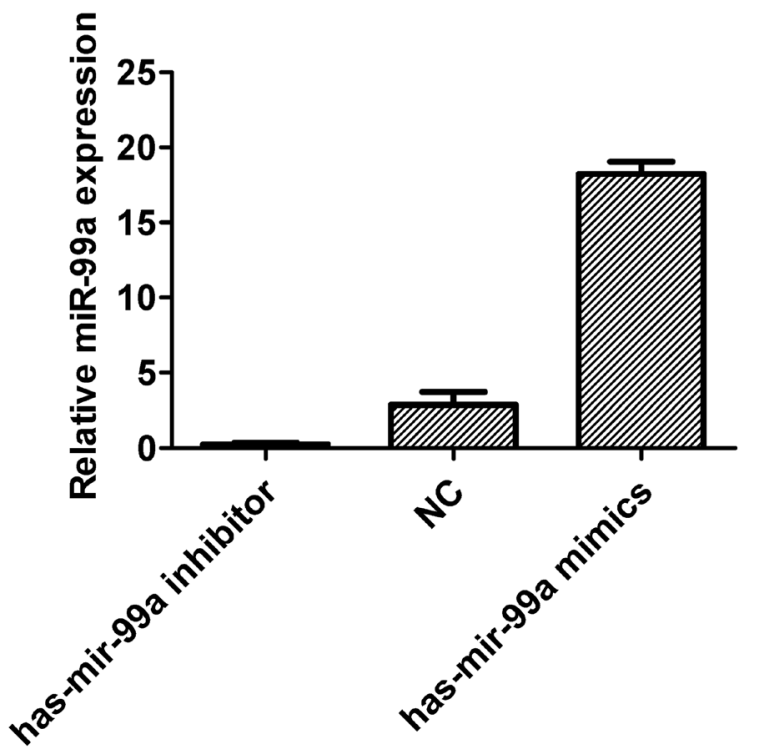

Figure 2. Relative miR-99a expressions after transfection detected by RT-PCR.

became more obvious with prolonged time. In contrast, the hsa-miR-99a inhibitor group grew significantly faster than the NC group did. Collectively, up-regulating miR-99a inhibited the proliferation of glioma cells, but knocking this gene down promoted their proliferation.

\section{Cell migration detected by scratch assay}

The migration capacity of tumor cells is a crucial index reflecting malignant phenotypes, which was herein detected by the scratch assay. As evidenced by the width or acellular area of scratch at $0 \mathrm{~h}$ and $24 \mathrm{~h}$, the migration capacities of hsa-miR-99a mimics and hsa-miR-99a inhibitor groups significantly decreased and increased respectively compared with that of the NC group (Figure 4). Taken together, up-regulating miR99a suppressed the migration of glioma cells, whereas knocking this gene down facilitated their migration.

\section{Discussion}

With a morbidity rate of approximately 5-8/0.1 million, malignant gliomas are refractory and prone to relapse due to invasive growth [10]. At present, intracranial malignant gliomas are mainly treated by surgeries alone or in combination with radiotherapy and chemotherapy when necessary. However, glioma tissues, especially glioblastoma ones, can hardly be differentiated from surrounding normal tissues only by surgeries owing to high invasion and migration capacities, leading to high recurrence rate [11]. Moreover, tumor cells cannot be specifically killed by chemotherapy or radiotherapy, also inducing a series of toxic side reactions. Thus, the prognosis is often poor, which requires study on the biological processes of glioma progression and development of novel therapies in order to
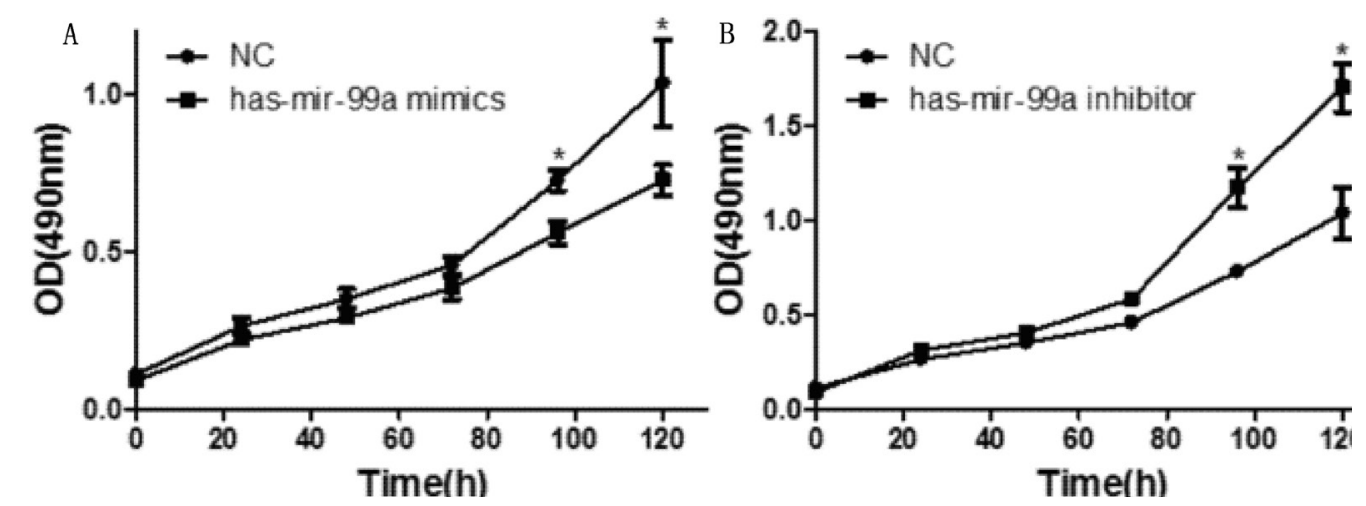

Figure 3. MiR-99a significantly inhibited LN229 cell proliferation. A: Proliferation curve of miR-99a upregulation group; B: proliferation curve of miR-99a knock-down group. 
A

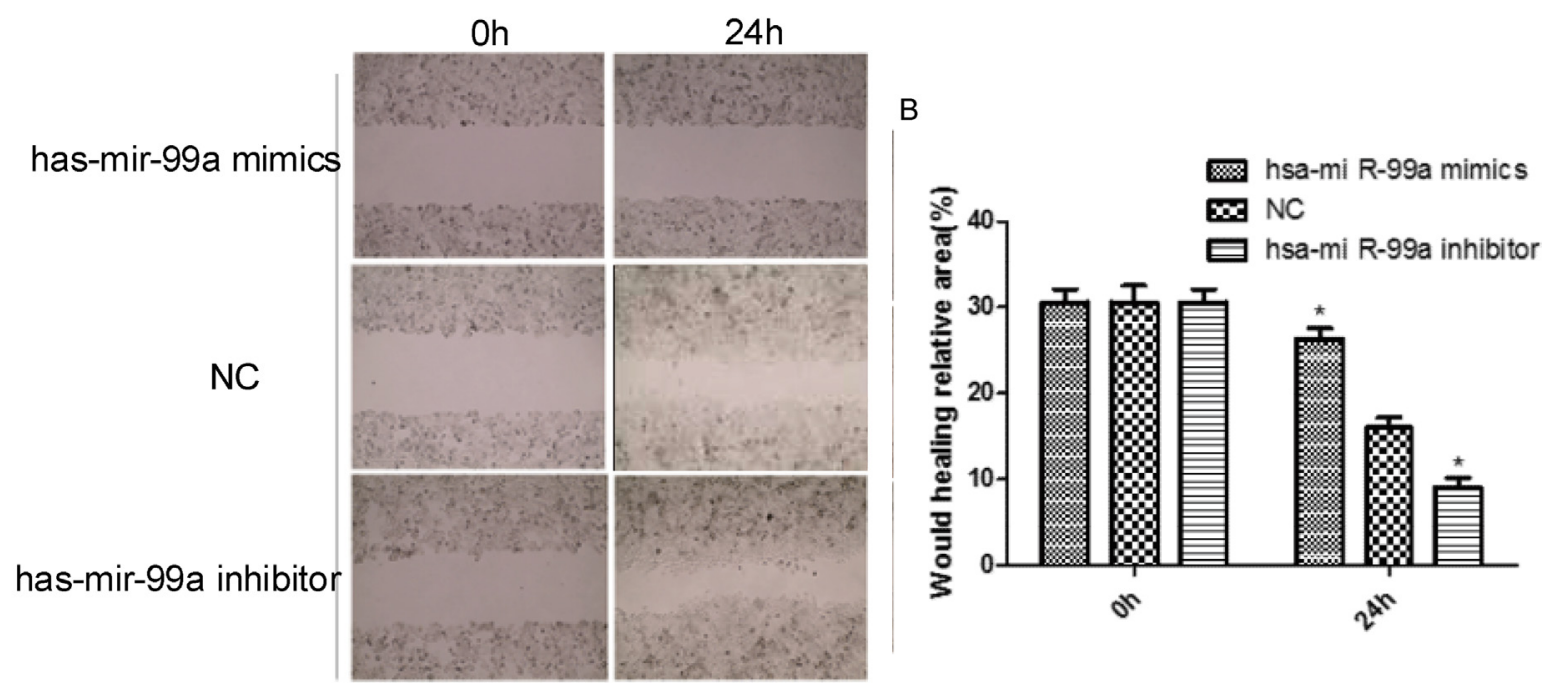

Figure 4. LN229 cell migration detected by scratch assay.

prolong the survival of patients and to improve the treatment outcomes [12,13]. Recently, gene therapy has been employed to treat gliomas. By using human menstrual blood-derived MSCs as a gene delivery vehicle, Wang et al. developed an effective adenoviral serotype 35 transduction system which targeted the delivery of secretable trimeric TRAIL to glioma sites specifically [14]. Also, Portnow et al. treated recurrent high-grade glioma patients through intracranial administration of human neural stem cells stably expressing cytosine deaminase, with effective and stable outcomes [15].

Notably, miRNAs have been involved in modulating the apoptosis and autophagy of malignant glioma, providing new potential therapeutic targets [16]. In mammals, microRNA regulates gene expression by degrading target mRNA and suppressing the translation process through base-pairing with the 3'-untranslated region [17]. Particularly, miR-99a is a welldocumented anti-oncogene. Yu et al. found that miR-99a played a crucial role in the tumorigenesis of non-small cell lung cancer, probably as a therapeutic target for preventing the metastasis of tumor cells [18]. By suppressing E2F2 and
EMR2, miR-99a has been reported to inhibit the in vivo epithelial-to-mesenchymal transition of epithelial cells, accompanied by decreased number of cancer stem cells [19]. Nevertheless, the relationship of miR-99a with glioma is still largely unknown. Thereby motivated, we herein constructed LN229 cells with stable up-regulation or knock-down of miR-99a, and studied the changes of their proliferation and migration.

It has recently been reported that miR-99a may regulate tumor cell proliferation through mTOR, AKT1 and FGFR3 [20-22], but detailed studies on the mechanisms of action remain lacking. In this study, MTT assay showed that up-regulating miR-99a inhibited the proliferation of glioma cells, but knocking it down promoted their proliferation. Additionally, scratch assay exhibited that up-regulation of miR-99a suppressed the migration of glioma cells, whereas knock-down of this gene facilitated their migration. Similarly, Zhang et al. detected miR99a expression levels in glioma tissues by using quantitative real-time PCR [23], which significantly reduced and were closely associated with tumor stage, further verifying that miR-99a was a feasible target for glioma therapy. 
In summary, up-regulating miR-99a attenuated the proliferation and migration capacities of glioma cells LN229, whereas knocking it down enhanced their proliferation and migration. Hence, miR-99a indeed significantly affected the proliferation and migration capacities of glioma cells, as a potentially eligible therapeutic target for this tumor. Nevertheless, this study still has some limitations. First, we only selected one type of glioma cell line (LN229), and thus will test more cell lines to validate these results. Second, further experiments using animals and human subjects are needed to prove the effects of miR-99a on the migration and proliferation of glioma cells.

\section{Conflict of interest}

There was no conflict in this work.

\section{Acknowledgement}

This study was financially supported by Natural Science Foundation of China (No. 81171153).

\section{Abbreviations}

miRs $=$ microRNAs

FBS $=$ Fetal bovine serum

\section{References}

1. Ma CC, Xiong Z, Zhu GN, Wang C, Zong G, Wang $\mathrm{HL}$, et al. Long non-coding RNA ATB promotes glioma malignancy by negatively regulating miR-200a. J Exp Clin Cancer Res. 2016;35(1):90. DOI: 10.1186/s13046016-0367-2

2. Cui B, Li B, Liu Q, Cui Y. lncRNA CCAT1 promotes glioma tumorigenesis by sponging miR-181b. J Cell Biochem. 2017;118(12):4548-57. DOI: 10.1002/ jcb.26116

3. Yin D, He X, Zhang E, Kong R, De W, Zhang Z. Long noncoding RNA GAS5 affects cell proliferation and predicts a poor prognosis in patients with colorectal cancer. Med Oncol. 2014;31(11):253. DOI: 10.1007/ s12032-014-0253-8
4. Chen C, Zhao Z, Liu Y, Mu D. microRNA-99a is downregulated and promotes proliferation, migration and invasion in non-small cell lung cancer A549 and H1299 cells. Oncol Lett. 2015;9(3):1128-34. DOI: 10.3892/ ol.2015.2873

5. Zhang J, Jin H, Liu H, Lv S, Wang B, Wang R, et al. MiRNA-99a directly regulates AGO2 through translational repression in hepatocellular carcinoma. Oncogenesis. 2014;3:e97. DOI: 10.1038/oncsis.2014.11

6. Feng Y, Kang Y, He Y, Liu J, Liang B, Yang P, et al. microRNA-99a acts as a tumor suppressor and is down-regulated in bladder cancer. BMC Urol 2014;14(1):50. DOI: 10.1186/1471-2490-14-50

7. Sun M, Hong S, Li W, Wang P, You J, Zhang X, et al. miR-99a regulates ROS-mediated invasion and migration of lung adenocarcinoma cells by targeting NOX4. Oncol Rep. 2016;35(5):2755-66. DOI: 10.3892/ or.2016.4672

8. Hao B, Cui L, Gu Y, Zhang B, Wang M, Zhou H, et al. MiR-99A Suppresses Proliferation, Migration, Invasion and Induces G1-Phase Cell Cycle Arrest via Targeting IGF-1R Pathway in Renal Cell Carcinoma 786-0 and OS-RC-2 Cells. Urology. 2017;doi: 10.1016/j.urology.2017.01.003. DOI: 10.1016/j.urology.2017.01.003

9. Parker BC, Annala MJ, Cogdell DE, Granberg KJ, Sun $\mathrm{Y}$, Ji P, et al. The tumorigenic FGFR3-TACC3 gene fusion escapes miR-99a regulation in glioblastoma. J Clin Invest. 2013;123(2):855-65. DOI: 10.1172/JCI67144

10. Barbagallo D, Condorelli A, Ragusa M, Salito L, Sammito M, Banelli B, et al. Dysregulated miR-671-5p/ CDR1-AS/CDR1/VSNL1 axis is involved in glioblastoma multiforme. Oncotarget. 2016;7(4):4746-59. DOI: 10.18632/oncotarget.6621

11. Li X, Liu Y, Granberg KJ, Wang Q, Moore LM, Ji P, et al. Two mature products of MIR-491 coordinate to suppress key cancer hallmarks in glioblastoma. Oncogene. 2015;34(13):1619-28. DOI: 10.1038/onc.2014.98

12. Capelle L, Fontaine D, Mandonnet E, Taillandier L, Golmard JL, Bauchet L, et al. Spontaneous and therapeutic prognostic factors in adult hemispheric World Health Organization Grade II gliomas: a series of 1097 cases. J Neurosurg. 2013;118(6):1157-68. DOI: 10.3171/2013.1.JNS121

13. Chen YS, Chen ZP. Vasculogenic mimicry: a novel target for glioma therapy. Chin J Cancer. 2014;33(2):74-9. DOI: $10.5732 /$ cjc.012.10292

14. Wang XJ, Xiang BY, Ding YH, Chen L, Zou H, Mou 
$\mathrm{XZ}$, et al. Human menstrual blood-derived mesenchymal stem cells as a cellular vehicle for malignant glioma gene therapy. Oncotarget. 2017;8(35):58309-21. DOI: 10.18632 /oncotarget.17621

15. Portnow J, Synold TW, Badie B, Tirughana R, Lacey SF, D'Apuzzo M, et al. Neural Stem Cell-Based Anticancer Gene Therapy: A First-in-Human Study in Recurrent High-Grade Glioma Patients. Clin Cancer Res. 2017;23(12):2951-60. DOI: 10.1158/1078-0432.CCR16-1518

16. Palumbo S, Miracco C, Pirtoli L, Comincini S. Emerging roles of microRNA in modulating celldeath processes in malignant glioma. J Cell Physiol. 2014;229(3):277-86. DOI: 10.1002/jcp.24446

17. Gerresheim GK, Dünnes N, Nieder-Röhrmann A, Shalamova LA, Fricke M, Hofacker I, et al. microRNA-122 target sites in the hepatitis C virus RNA NS5B coding region and 3' untranslated region: function in replication and influence of RNA secondary structure. Cell Mol Life Sci. 2017;74(4):747-60. DOI: 10.1007/ s00018-016-2377-9

18. Yu SH, Zhang CL, Dong FS, Zhang YM. miR-99a suppresses the metastasis of human non-small cell lung cancer cells by targeting AKT1 signaling pathway. J Cell Biochem. 2015;116(2):268-76. DOI: 10.1002/ jcb. 24965

19. Feliciano A, Garcia-Mayea Y, Jubierre L, Mir C, Hummel M, Castellvi J, et al. miR-99a reveals two novel oncogenic proteins E2F2 and EMR2 and represses stemness in lung cancer. Cell Death Dis. 2017;8(10):e3141. DOI: $10.1038 /$ cddis.2017.544

20. Modi BP, Washington S, Walsh SW, Jackson-Cook C, Archer KJ, Strauss JF 3rd. Expression patterns of the chromosome 21 MicroRNA cluster (miR-99a, miR$125 \mathrm{~b}$ and let-7c) in chorioamniotic membranes. Placenta. 2017;49:1-9. DOI: 10.1016/j.placenta.2016.11.002

21. Cheng H, Xue J, Yang S, Chen Y, Wang Y, Zhu Y, et al. Co-targeting of IGF1R/mTOR pathway by miR497 and miR-99a impairs hepatocellular carcinoma development. Oncotarget. 2017;8(29):47984-97. DOI: 10.18632/oncotarget.18207

22. Mei LL, Qiu YT, Huang MB, Wang WJ, Bai J, Shi ZZ. MiR-99a suppresses proliferation, migration and invasion of esophageal squamous cell carcinoma cells through inhibiting the IGF1R signaling pathway. Cancer Biomark. 2017;doi: 10.3233/CBM-170345. DOI: 10.3233/CBM-170345

23. Zhang M, Guo Y, Wu J, Chen F, Dai Z, Fan S, et al. Roles of microrna-99 family in human glioma. Onco Targets Ther. 2016;9:3613-9. 